\title{
The New Autonomous Incentive Hardware Platform Indoor of Automotive ABS System
}

\author{
Yue Liang ${ }^{\text {a }}$ \\ Hunan Defense Industry Polytechnic, 411207, Xiangtan, China \\ a719178441@qq.com
}

Keywords: ABS, solenoid valve, dynamic signal, independent excitation

\begin{abstract}
The single-chip microcomputer generates a 4-channel square wave input to the ABS-driven solenoid valve, it drives the continuous operation of the ABS system, which lays a foundation for the ABS system fault diagnosis research. It established a data acquisition system that collected vibration signals, sound signals, and pressure signals, and these signals can correspond to the opening process of the solenoid valve in time, and analyze and process, monitor and extract the characteristic parameters of the dynamic signal. This can reflect the mechanical movement of the solenoid valve and enable the detection of the failure of the ABS system.
\end{abstract}

\section{Introduction}

The driver quickly brakes in an emergency and causes the wheels to lock up. The lateral adhesion between the wheel and the road surface will completely disappear. If the front wheel is locked and the front wheel is still rolling, the car will lose its ability to turn; if the rear wheel is locked and the front wheel is still rolling, the car will slide (tail flick), both of these situations are very likely to cause some serious accidents. Anti-lock braking system (ABS) can automatically adjust the brake force of the wheel to prevent the wheel from locking when braking and achieve the best braking effect of the braking system. The ABS system can effectively shorten the braking distance, improve the stability and maneuverability of the direction, and has important significance to the driving safety of the vehicle. Therefore, the fault detection of the ABS system is also receiving more and more attention. At present, regarding the detection of the failure of the ABS system in the world, the main method is the road vehicle test and the indoor construction test stand. Because the pavement test of ABS system must be on some special roads, the required site area must be large and expensive; The testing equipment that needs to be used in the test, the preparation required for the test and the entire test process take a long time; The ABS system can only be opened by emergency braking at high speed, so the risk of the actual vehicle test is extremely high; The test of the ABS system is easily affected by the environment, and the reproducibility of the data is difficult to analyze. Therefore, the road test of the ABS system can only be applied to a certain type of automobile fitting test or partial sampling. It is not suitable for regular inspection of a large number of automobiles. Therefore, more scholars and researchers have turned their attention to the indoor test bench. 


\section{The Dynamically Stimulated Signal Simulation}

Because the wheel speed signal itself is irregular, it is difficult to simulate and control. When the wheel speed signal is input to the ABS system, the collected data is very irregular and it is difficult to find the comparability of dynamic data. Therefore, a square wave signal that is relatively simple and easy to control is selected as the control signal.

That is, a square wave signal is edited in MATLAB/Simulink. The simulation block diagram is shown in Figure 1. Its parameter settings include amplitude, period, pulse width, phase delay, and control signals of the ABS system — Square wave signal output DAC 1 and DAC 3, one motor control signal output DAC 7. After Matlab's real-time interface is downloaded to the amplifier circuit board and motor drive module, respectively, and then control the ABS system work.

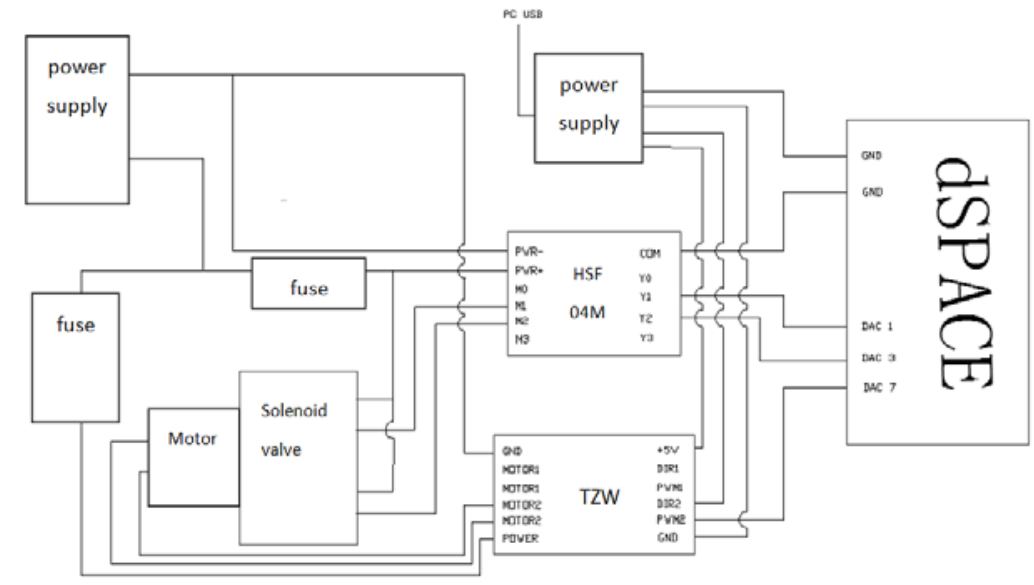

Figure 1. The circuit connection of the hardware platform

The hardware platform selects this simple square wave signal as the input signal, mainly taking into account the technical blockade of the ABS system, the ABS control strategy is unknown, especially the control strategies of different types of ABS systems are also different. Therefore, bypassing the control strategy of the ABS system and directly detecting and analyzing the entire ABS is the starting point for the research of this experimental platform. When a simple square wave signal is used as the input signal, the dynamic signal change is relatively simple, which is easy to control and find some problems, and the change of the ABS system's fault is also more obvious, so that it is easier to build a fault database. This set the direction for the mechanical fault diagnosis of ABS systems.

\section{Design of Dynamic Excitation Hardware Test Bench for ABS System}

The interface definition of the total achievement of the ABS system is the input signal type corresponding to each interface, so the total achievement of the ABS system is an important way to verify the driving results. A square signal output from dSPACE is input to the inlet and outlet valve interfaces of the ABS system assembly through the corresponding DAC1 and DAC 3 interface definitions. This can control the activation and deactivation of the solenoid valve of an ABS system. After Simulink's model was automatically generated by Matlab, the code was successfully downloaded into dSPACE's hardware MicroAutoBox, and entered into the ABS system's assembly by the corresponding interface definition, then the solenoid valve of the ABS system starts to work, and at this time, a person can clearly hear the sound of the solenoid valve of the ABS system.

In this drive system, the hardware design is mainly divided into five parts: the signal generation part, the power supply part, the signal amplification part, the ABS system brake power pedestal, and the motor drive part. The hardware part is mainly completed on the ABS system brake training 
platform. The training platform has complete braking system required for the experiment, including the master cylinder, brake line, brake pedal, etc. It can semi-simulate the real vehicle state, which improves the accuracy and practicality of the experimental data. The indoor hardware test bench can study the fault condition of the ABS system. Through the collection of sound signals, vibration signals and pressure signals, the difference between the ABS fault-free status and the fault status is compared and analyzed, and the law is summarized, and the cause of the failure of the ABS system is explored.

\subsection{HSF04M 4-channel PLC Output Amplifier Board}

HSF04M 4-channel PLC output amplifier board with optical isolation, not only can reliably protect the output contacts of the PLC, but also can work stably under severe working environment. HSF04M is used to expand the output of common anode transistor output or relay output type PLC with load capacity, and can drive solenoid valve with 6-16 ports and low power DC motor within 75W.

\subsection{TZW DC Motor Driver Board}

TZW is an industrial-grade 15A dual DC motor drive module, H-bridge, high power, with a brake function. With two motor interfaces and a signal input interface, $5 \mathrm{~V}$ power is required to power it. The driver consists of a full two half-bridge driver IC and a very low internal resistance N-channel MOSFET. The switching loss of the MOSFET is minimized, improving the power supply utilization. Because the MOSFET driver chip hardware brake function and power feedback function, The MOSFET uses an N-channel IRF3205 MOSFET and uses two dedicated half-bridge driver chips. The top tube uses a bootstrap capacitor. This way, the upper tube has enough drive voltage to quickly turn on the MOSFET channel, which increases the acceleration of the motor. At the same time, it can also quickly brake the motor. This design is more able to meet the fast start-up and fast braking required in the solenoid valve operation of the ABS system. The driver can work in 0\%-99\% PWM modulation duty cycle, so that the motor can get enough drive voltage. Motor forward: $D I R=1 \mathrm{PWM}=\mathrm{PWM}$; motor reversal: DIR=0 PWM=PWM; parking brake: DIR=X PWM=0.

\section{The Experimental Process}

The test bench was set up according to the design diagram and the test bench was started according to the following procedure.

(1) Turn on the battery power switch, turn on dSPACE power switch, turn on the motor drive power switch, and you can see the indicator light.

(2) Click Matlab/Simulink on the computer to open the simulation model.

(3) Click Controldesk software to prepare to connect with Simulink.

(4) Open the parameter file. Run the Simulink model to see the results of the simulation model.

(5) Click on the Real-Time Workshop option in the Tools toolbar in Simulink, and then click Build Model. This operation activates the connection between Simulink and Controldesk. The simulation block diagram in Simulink is automatically compiled to generate the $\mathrm{C}$ language code.

(6) After the code is generated, the entire system is connected. Click the open button on the Controldesk interface to start the ABS system. At the same time, open the signal acquisition interface of the YE7600 dynamic data acquisition instrument and observe the dynamic signal acquisition process of the four channels.

(7) You can clearly hear the regular sound of the ABS system standing next to you, and the frequency of the input signal is the same. 
(8) The collected sound signals, vibration signals, and pressure signals are converted into Matlab files for later data processing and analysis.

(9) Man-made oil leakage failure on the pipeline, collecting corresponding sound signals, vibration signals, and pressure signals.

(10) Artificial analog electromagnetic valve clamping failure on the pipeline, collecting the corresponding sound signal, vibration signal, pressure signal.

(11) Analysis of late-stage data. Corresponding data processing and analysis are performed on the collected dynamic signals to arrive at a conclusion.

\section{Conclusion}

Through the ABS drive system jointly developed by dSpace and MATLAB/Simulink, this successfully achieved the continuous operation of the ABS solenoid valve under the premise of being out of the car, which satisfies the actual requirements of the indoor ABS system experiment. A dynamic excitation simulation square wave is used to simulate the wheel speed signal, and the ABS valve is continuously actuated by hardware to realize the function of the ABS controller.

\section{Acknowledgments}

This work was financially supported by Hunan Defense Industry Polytechnic, General Issues, Research on Dynamic Signal Detection System of Auto ABS System Braking Platform (Project number: 2017YJYBKT08)

\section{References}

[1] Zhan-qun Shi, Gu F, Ball A. Prediction of Anti-Lock Braking System Condition with Vehicle Stationary Using Model-based Approach, International Journal of Automotive Technology, 2010,11 (3):363-373.

[2] Yue LIANG, Shuo YANG, Yong-Jian WANG, Dong ZHEN, Zhan-qun SHI, A Simulation Study On the ABS System Based on the Threshold Controlling,2015 International Conference on Mechanical Science and Mechanical Design(MSMD2015),2015.12.12-13

[3] Dressler O, Struss P. A toolbox integrating model-based diagnosability analysis and automated generation of diagnostics [J]. Proceedings of International Workshop on Principles of Diagnosis, 2003.

[4] Isermann I H C R, Münchhof I M. Application Examples[M]. Springer Berlin Heidelberg,2011.

[5] Straky H, Kochem M, Schmitt J, et al. Influences of braking system faults on vehicle dynamics[J]. Control Engineering Practice, 2003,11(3):337-343. 